\title{
Sho-saiko-to, a traditional herbal medicine, regulates gene expression and biological function by way of microRNAs in primary mouse hepatocytes
}

Kwang Hoon Song ${ }^{1,3}$, Yun Hee Kim² and Bu-Yeo Kim²

\begin{abstract}
Background: Sho-saiko-to (SST) (also known as so-shi-ho-tang or xiao-chai-hu-tang) has been widely prescribed for chronic liver diseases in traditional Oriental medicine. Despite the substantial amount of clinical evidence for SST, its molecular mechanism has not been clearly identified at a genome-wide level.

Methods: By using a microarray, we analyzed the temporal changes of messenger RNA (mRNA) and microRNA expression in primary mouse hepatocytes after SST treatment. The pattern of genes regulated by SST was identified by using time-series microarray analysis. The biological function of genes was measured by pathway analysis. For the identification of the exact targets of the microRNAs, a permutation-based correlation method was implemented in which the temporal expression of mRNAs and microRNAs were integrated. The similarity of the promoter structure between temporally regulated genes was measured by analyzing the transcription factor binding sites in the promoter region.

Results: The SST-regulated gene expression had two major patterns: (1) a temporally up-regulated pattern (463 genes) and (2) a temporally down-regulated pattern (177 genes). The integration of the genes and microRNA demonstrated that 155 genes could be the targets of microRNAs from the temporally up-regulated pattern and 19 genes could be the targets of microRNAs from the temporally down-regulated pattern. The temporally up-regulated pattern by SST was associated with signaling pathways such as the cell cycle pathway, whereas the temporally down-regulated pattern included drug metabolism-related pathways and immune-related pathways. All these pathways could be possibly associated with liver regenerative activity of SST. Genes targeted by microRNA were moreover associated with different biological pathways from the genes not targeted by microRNA. An analysis of promoter similarity indicated that co-expressed genes after SST treatment were clustered into subgroups, depending on the temporal expression patterns.

Conclusions: We are the first to identify that SST regulates temporal gene expression by way of microRNA. MicroRNA targets and non-microRNA targets moreover have different biological roles. This functional segregation by microRNA would be critical for the elucidation of the molecular activities of SST.
\end{abstract}

Keywords: Sho-saiko-to, Microarray, MicroRNA, Hepatocyte, Pathway, Promoter

\footnotetext{
* Correspondence: buykim@kiom.re.kr

${ }^{2}$ Herbal Medicine Research Division, Korea Institute of Oriental Medicine,

1672 Yuseongdae-ro, Yuseong-gu, Daejeon 305-811, Republic of Korea

Full list of author information is available at the end of the article
} 


\section{Background}

Sho-saiko-to (SST) (also known as so-shi-ho-tang or xiaochai-hu-tang) is a botanical formulation composed of seven herbal materials (see Additional file 1: Table S1) and is widely used for the treatment of chronic hepatitis and liver cirrhosis in Korea, Japan, and China [1]. SST and its major components (e.g., baicalin, baicalein, glycyrrhizin, and saikosaponinD) have marked antiproliferative activity on hepatocellular carcinoma [2-4], prevent liver injury [5], and promote liver regeneration in animal models [6,7]. These pharmacologic effects of SST involve the immunomodulation of diverse immune cells and immune molecules [8,9]. However, because of the complex nature of the chemical components of SST, focusing only on specific components or on a few target genes is inadequate to understand the diverse biological activities of SST. Therefore, it is necessary to apply a multiple target-based approach to elucidate the molecular mechanisms mediated by the multiple components of SST.

Recent advances in high-throughput technology such as the microarray has made it possible to investigate the effects of drugs at the whole-genome level [10]. One highthroughput technology is the microRNA array, which can detect the expression level of whole microRNAs that have been discovered to date [11]. MicroRNA is a small noncoding RNA molecule composed of approximately 22 nucleotides that pair to sites in messenger RNA (mRNA) and directly repress post-transcription in eukaryotic cells [12]. Many reports suggest that microRNAs are involved in diverse biological functions such as proliferation, differentiation, and development. The search for targets of microRNA shows that many mammalian mRNAs are the conserved targets of microRNA [13]. This suggests an important role of microRNA in regulating gene expression. Therefore, using the information of mRNA and microRNA is important to elucidate the precise mechanism of gene expression. The integrated multi-omics approach actually reveals a novel regulatory network of gene expression in diverse biological situations such as disease research [14-16], genome research [17], and herbal research $[18,19]$. We also previously reported the usefulness of a genome-wide approach in elucidating the molecular effects of herbal extracts [20,21].

By using an integrated genomic analysis of genes and microRNAs in the present study, we attempted to identify SST-induced gene expression changes in primary mouse hepatocytes. The results indicated that SST regulated gene expression through microRNA in a functionally coordinated manner. Our approach could give perspective on the role of microRNAs in the pharmacological effects of SST.

\section{Methods}

Primary mouse hepatocyte isolation and culture

Six-week-old male ICR mice were purchased from Samtako Bio Inc. (Seoul, Korea). Primary mouse hepatocytes were prepared by using the collagenase perfusion method. In brief, the 6-week-old male mice were anesthetized by an intraperitoneal injection of Zoletil-50 and 2\% Rompun, which were cannulated through the right ventricle. The livers were perfused with ethylene glycol tetra-acetic acid (0.5 mM) in Hepes-buffered Hank's balanced salt solution (HBSS; $\mathrm{pH} 7.4$ ) for $5-6 \mathrm{~min}$ (flow rate $5 \mathrm{~mL} / \mathrm{min}$ ). The livers were then perfused for another $20 \mathrm{~min}$ with Hepesbuffered HBSS containing collagenase (Sigma, USA) (flow rate $5 \mathrm{~mL} / \mathrm{min}$ ). The hepatocytes were dispersed, washed, and purified on a Percoll density gradient (Sigma). Hepatocyte preparations with viability greater than $85 \%$, as determined by the trypan blue exclusion protocol, were used. The isolated hepatocytes were suspended, and then transferred to gelatin-coated culture dishes or plates at a density of approximately $5 \times 10^{5}$ cells $/ \mathrm{mL}$. The hepatocytes were allowed to attach onto culture dishes or plates coated with gelatin for 4-6 hours in William's Media E (Sigma) containing $1 \%$ penicillin/streptomycin, $2 \mathrm{mM}$ of L-glutamine and $10 \%$ fetal bovine serum. After the attachment, the hepatocytes were washed with HBSS and provided fresh medium. They were incubated overnight at $37^{\circ} \mathrm{C}, 95 \%$ air, and $5 \%$ carbon dioxide. The hepatocytes were then deprived of the serum and used for experiments. All animal experimental procedures were approved by Institutional Animal Care and Use Committee of the Korea Institute of Oriental Medicine (Permit Number: KIOM 12-024) and performed in strict accordance with the recommendations in the Guide for the Care and Use of Laboratory Animals at the Korea Institute of Oriental Medicine.

\section{Cell viability assay}

SST was kindly provided by Dr. Hyeun Kyoo Shin (Basic Herbal Medicine Research Group, Korea Institute of Oriental Medicine, Republic of Korea). Preparation of SST was described as previously [22]. In brief, crude seven herbal medicines were extracted in distilled water at $100^{\circ} \mathrm{C}$ for 2 hours, filtered, and then freeze-dried. We confirmed the safety of SST by using an in vitro colorimetric cell proliferation kit (methyl thiazolyl tetrazoliym [MTT]) (Roche Applied Science, Germany) as described previously [23]. In brief, hepatocytes were first cultured in 48-well plates at a density of $1.0 \times 10^{5}$ cells/well for 24 hours. After incubation, the cells were washed with phosphate-buffered saline and treated with different concentrations of SST $(0.1-1.0 \mathrm{mg} / \mathrm{mL})$ for 24 hours. The cells were hereafter washed and incubated for 1 hour with MTT $(500 \mu \mathrm{g} / \mathrm{mL})$. Formazan crystals were dissolved by using dimethyl sulfoxide (100 $\mu \mathrm{L} /$ well). The absorbance was measured colorimetrically at $570 \mathrm{~nm}$.

\section{Microarray experiment and quantitative real-time polymerase chain reaction}

Mouse primary hepatocytes were treated with $500 \mu \mathrm{g} / \mathrm{mL}$ of SST at a density of $1.0 \times 10^{6}$ cells per 60 -mm dish for 
1-24 hours in triplication. The total RNA from hepatocytes was isolated with Tri-reagent (Sigma) in accordance with the manufacturer's instructions. The quality of purified RNA was measured by using the Agilent 2100 Bioanalyzer (Agilent Technologies, USA); only samples with a RNA integrity number (RIN) greater than 7.0 were included in the microarray analysis. RNAs from the triplication of experiments at each time point were pooled to exclude experimental bias. For the gene expression microarray, isolated RNA was amplified and labeled by using the low RNA input linear amplification Kit PLUS and then hybridized to a microarray (Agilent Mouse Whole Genome $44 \mathrm{~K}$; Agilent Technologies, USA) that contained approximately 44,000 probes (approximately 26,600 unique genes) in accordance with the manufacturer's instructions. For microRNA expression microarray, the microRNA was labeled and hybridized to Agilent Mouse miRNA Microarray (Release 17.0) by using the Agilent miRNA Labeling and Hyb Kit (Agilent Technologies, USA). Approximately 1100 microRNAs, based on the annotation of miRBase Release 17.0, were presented in microarray. The arrays were then scanned with the Agilent Microarray Scanner (Agilent Technologies, USA). For quantitative real-time polymerase chain reaction (Q-PCR) analysis, $\mathrm{mRNA}$ and microRNA were reverse-transcribed, amplified, and detected by using Taqman probes (ABI, USA) in triple time, as described previously [24].

\section{Microarray data analysis}

The raw intensity of the probe signals was obtained by using Feature Extraction Software (Agilent Technologies, USA). Only array elements showing a signal intensity greater than 1.4-fold of the local background were considered well measured. The remaining elements were normalized using the quantile method [25]. The intensities for duplicated spots were averaged. The expression ratio of genes (or microRNAs) in the experimental samples was then determined by comparing them with genes (or microRNAs) in the control sample. The expression profile was hierarchically clustered by using the Cluster program and visualized using the TreeView program (both can be obtained from http://www.eisenlab.org). Figure 1 shows a schematic diagram of the overall analytical process.

\section{Temporal expression of genes and microRNAs}

The short time-series expression miner (STEM) program-which was originally developed for the temporal analysis of microarray experiments [26] —was used to identify genes or expression patterns that were changed temporally. Only genes with a fold ratio greater than 2 or less than 0.5 for at least one time point were included in the analysis. The statistical significance of the temporal patterns was calculated by using a permutation test $(n=1000)$ corrected by the false discovery rate (FDR).

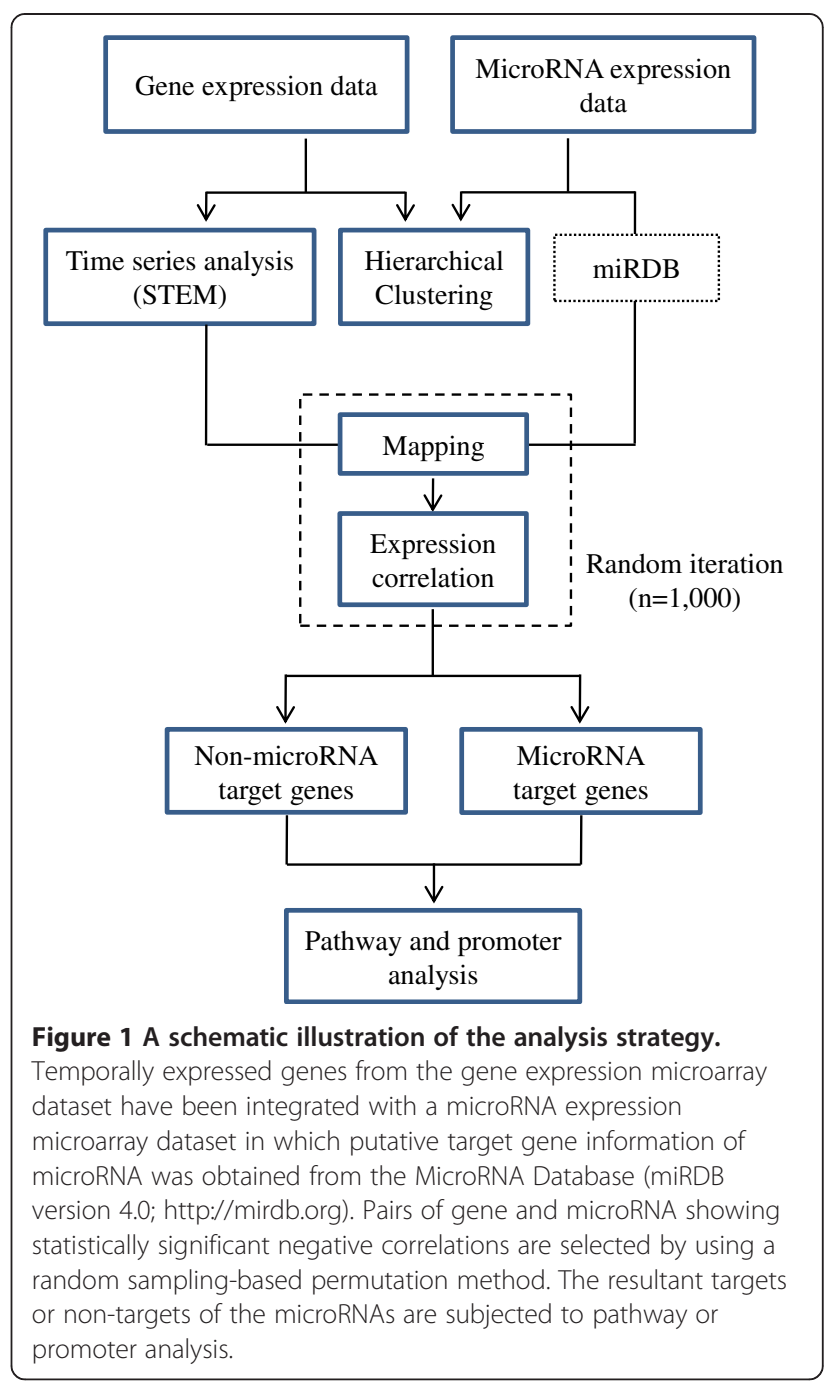

\section{Integration of mRNA and microRNA expression}

The relationship between gene expression and microRNA expression was measured by using a permutationbased correlation method. First, a list of the predicted target genes of microRNAs, calculated by bioinformatic analysis of large public microarray datasets, was obtained from the MicroRNA Database (miRDB version 4.0) website (http://mirdb.org) [27,28]. Second, the Pearson correlation coefficient was measured between each microRNA expression in the microRNA microarray and each predicted target gene expression in the mRNA microarray. Only gene and microRNA pairs that showed a negative correlation coefficient were selected to form a correlation coefficient matrix between the predicted target genes and the microRNAs. The statistical significance of the resultant correlation coefficient matrix was estimated by using a random sampling-based permutation [29] in which the coefficient values from the original dataset were compared with the values from 1000 times randomly permuted datasets. Only target genes 
and microRNAs with a FDR less than 0.01 were selected as significant.

\section{Pathway enrichment}

The simple enriched pathways were estimated by the DAVID program [30] in which the $p$ values of each pathway were calculated, based on Fisher's exact test, from an input list of genes. For adjustment by multiple comparisons, the DAVID program used the FDR by the Benjamini procedure. For another pathway analysis, the Signaling Pathway Impact Analysis (SPIA) program [31] was implemented by using a subgroup of differentially expressed genes. The SPIA program calculated a global pathway significance $p$ value $\left(\mathrm{P}_{\mathrm{G}}\right)$ that combines the enrichment $p$ values and the perturbation $p$ values by considering pathway topology with a random bootstrap iteration number of 3000 . The FDR of the pathways was measured by applying the Benjamini algorithm in SPIA. The pathway information was obtained from the database of the Kyoto Encyclopedia of Genes and Genomes (KEGG, http://www.genome.jp/kegg).

\section{Pathway activity}

The activity of the pathways was measured by linearly combining the logarithmic expression value of all genes in each pathway to account for the accumulative effect of small changes by many genes [32]. Statistical significance was measured by the FDR in which the original pathway's activity was compared with the randomly permutated activity values (1000 times). Pathways with a FDR less than 0.01 were selected as significant and then hierarchically clustered on the basis of similarity of activity values.

\section{Core microRNA targets from multiple pathways}

Core nodes (i.e., core genes) among multiple pathways were measured by implementing KEGGgraph R package (version 2.10) [33]. In brief, the core nodes were determined by calculating the relative betweenness centrality of nodes in which the number of ingoing and outgoing edges for each node was computed in the network structure of the multiple pathways. Nodes with a relative betweenness centrality greater than 0.01 were selected as the core microRNA targets.

\section{Transcription factors binding sites analysis}

Candidate binding sites for transcription factors in the promoter region were identified through sequence matching of the position weight matrix by implementing MotifDb $\mathrm{R}$ package (version 1.2.2, http://www.bioconductor.org/packages/2.12/bioc/html/MotifDb.html) [34]. A total of 329 position weight matrices for mouse transcription factors were used. Of these, 47 matrices were from the JASPAR database (http://jaspar.genereg.net)
[35,36] and 282 matrices were from the Universal PBM Resource for Oligonucleotide-Binding Evaluation (UniPROBE) database [37]. The nucleotide sequence of the promoter region of the gene $(-2000 \mathrm{bp}$ to $+500 \mathrm{bp}$ from the transcription start site) was obtained from the Mus musculus full genome, which was provided by the University of California, Santa Cruz (UCSC mm10 version). The presence of the transcription factor binding site (TFBS) within the promoter region of each gene was predicted by using the matchPWM algorithm in which a minimum score for counting a match was set at 90\% [36]. Based on the resultant frequency of the matrices of the TFBS, the similarity of genes was determined by using Jaccard's algorithm, which does not consider the absence of binding sites in two promoters as an indication of similarity [38]. Jaccard's algorithm is effective in the promoter clustering of genes, as we previously reported [21].

\section{Results}

Temporal pattern of genes and microRNA expression

The cytotoxic effect of SST on primary hepatocytes was not significant under the experimental condition (0.1$1.0 \mathrm{mg} / \mathrm{mL}$ ) as shows (see Additional file 1: Figure S1). The concentration of SST therefore chosen for the study was $500 \mu \mathrm{g} / \mathrm{mL}$ because of its solubility and cytotoxicity in the microarray analysis. The expression profiles of genes and microRNAs, regulated by the treatment of SST, were measured by using microarray analysis in primary mouse hepatocytes. Figure 1 depicts the overall analysis. The expression pattern of genes shows that 1166 genes were dramatically changed in their expression levels at the time of SST treatment (Figure 2A). Among these patterns of gene expression, Sub-cluster 1 was composed of genes that temporally increased expression, whereas Sub-cluster 2 was composed of genes with temporally decreased expression. For a more systematic approach, we tried to isolate genes showing a specific temporal pattern by using a time-series analysis of the microarray. Figure $2 \mathrm{~B}$ presents two representative statistically significant temporal patterns: the temporal up-regulated pattern (temporal up-pattern) and the temporal down-regulated (temporal down-pattern); the FDR was less than 0.001 , which included most temporally expressed genes that were changed by SST. The temporal up-pattern included 463 temporally up-regulated genes and the temporal down-pattern included 177 genes down-regulated by SST. However, the expression of microRNAs did not show a clear temporal pattern after treatment with SST (Figure 2C) (see Additional file 1: Table S2) shows the full list of temporal pattern genes.

\section{Integration of gene and microRNA expression}

To determine the putative targets of microRNA among the genes in the two temporal expression patterns, we 


\section{(A)}

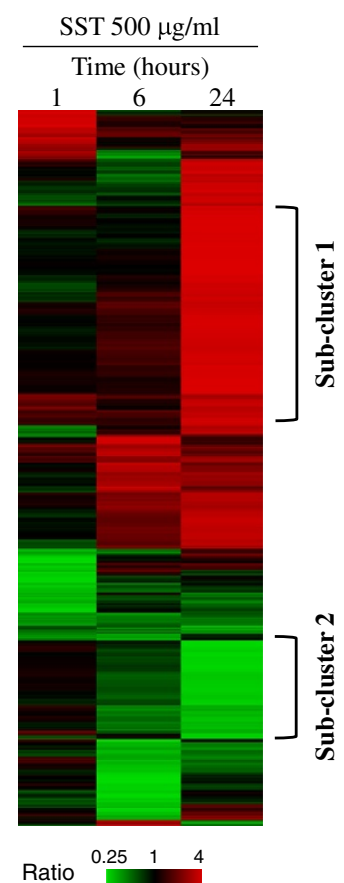

(B)
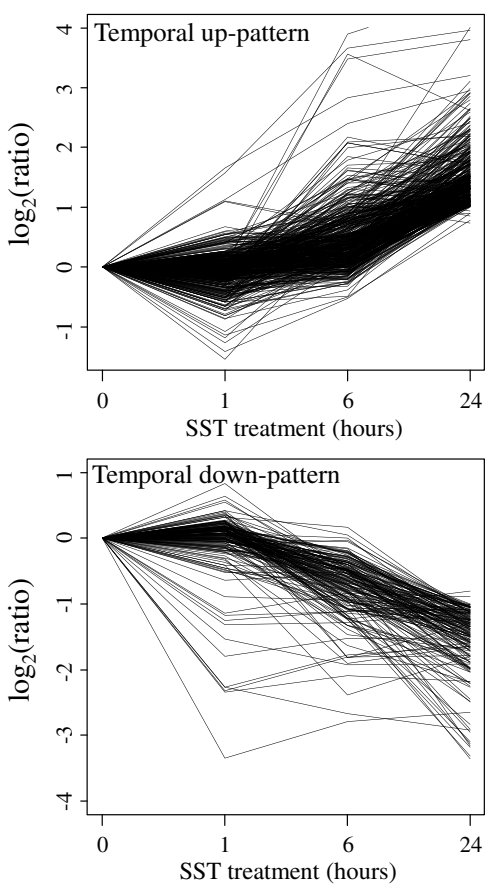

(C)

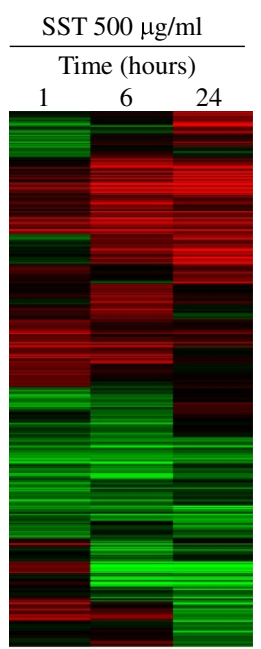

$\begin{array}{lll}0.5 & 0 & 2\end{array}$

Ratio

Figure 2 Temporal expression of genes and microRNAs after SST treatment in primary mouse hepatocytes. (A) Approximately 1100 differentially expressed genes with a fold ratio greater than 2 or less than 0.5 for at least one sample are clustered hierarchically. Sub-cluster 1 and Sub-cluster 2 indicate the two sub-clusters. Columns represent individual samples and rows represent individual genes. The expression ratio is represented in colors from red (i.e., high) to green (i.e., low), as indicated by the scale bar. (B) Temporally altered genes were identified by the Short Time-series Expression Miner (STEM) analysis and classified in two patterns (false discovery rate [FDR] less than 0.001). The temporal up-pattern comprises 463 genes and the temporal down-pattern comprises 177 genes. (C) Approximately 540 microRNAs with a minimum fold ratio of 1.5 in at least one sample are clustered hierarchically.

developed an algorithm integrating the temporal expressions of the genes and the microRNAs. By using the predicted target genes from the miRDB (http://mirdb.org) $[27,28]$, correlation coefficients were measured between the temporal expression of the predicted target genes and the microRNAs. After the permutation-based adjustment of the measured correlation coefficients, 174 genes with a FDR less than 0.01 were finally selected as the putative targets of microRNA from the two temporal patterns: 155 genes were identified from the temporal uppattern and 19 genes were identified from the temporal down-pattern. For experimental confirmation of the expression pattern based on microarray testing, Q-PCR was performed for some genes (ABCC4 from temporal up-pattern, CYP3A11 from temporal down-pattern, and FOXA1 from non-pattern) and microRNAs (miR-23a-3p and miR-466b$3 p)$. CYP3A11 was specifically the target of miR-23a-3p (Table 1). As shows (see Additional file 1: Figure S2), the overall patterns of gene expressions between microarray and Q-PCR were similar. Figure 3 shows the connection map between microRNA molecules and its target genes from temporal up-pattern (Figure 3A) and from temporal downpattern (Figure 3B). Table 1 lists the microRNA targets. The number of putative microRNA targets (19 of 177 genes) in the temporal down-pattern was significantly lower than the number of targets (155 of 463 genes) in the temporal uppattern ( $p$ value $<0.001)$. This unbalanced distribution of the microRNA target genes imply microRNAs have a specific biological role induced by SST. Therefore, we measured the functional involvement of microRNA targets via pathway analysis.

\section{Pathway analysis of putative microRNA targets}

The pathways involved in the two temporal patterns of the genes were measured by applying two different approaches (Table 2), by simple enrichment pathway analysis, and by topology-based signaling pathways analysis. Simple enrichment analysis of the pathways, which measures enriched pathways from Fisher's exact test, showed that the temporal up-pattern induced by SST was involved in the cell cycle pathway (i.e., KEGG ID mmu04110) and that the temporal down-pattern included drug metabolism-related pathways (e.g., mmu00982, mmu00983, mmu00980) and immunerelated pathways such as the systematic lupus erythematosus pathway (mmu05322) and the complement and coagulation cascade pathways (mmu04610). Topology-based signaling 
Table 1 The microRNA targets regulated by SST

\begin{tabular}{|c|c|c|c|c|c|c|c|c|}
\hline \multicolumn{9}{|c|}{ Temporal up-pattern } \\
\hline MicroRNA* & Target symbol & Target entrez & MicroRNA & Target symbol & Target entrez & MicroRNA & Target symbol & Target entrez \\
\hline \multirow[t]{12}{*}{ miR-495-3p } & Depdc1b & 218581 & miR-19b-2-5p & Sprr2a2 & $1 E+08$ & miR-3089-3p & Scara3 & 219151 \\
\hline & Slc1a2 & 20511 & & Mbnl3 & 171170 & & Rad51 & 19361 \\
\hline & Steap2 & 74051 & & Cep55 & 74107 & miR-3095-5p & Gsta3 & 14859 \\
\hline & Zmat1 & 215693 & & Tia1 & 21841 & & Cond1 & 12443 \\
\hline & Fmo5 & 14263 & miR-3092-5p & Cln6 & 76524 & miR-30c-5p & Fam43a & 224093 \\
\hline & Ckap4 & 216197 & & Lass3 & 545975 & & Fam49a & 76820 \\
\hline & $B c|2| 15$ & 229672 & & Gsto1 & 14873 & miR-322-3p & Ugdh & 22235 \\
\hline & Bst1 & 12182 & & C1qtnf1 & 56745 & & Mybl1 & 17864 \\
\hline & Pttg1 & 30939 & miR-450a-2-3p & Slc7a2 & 11988 & miR-343 & Mybl2 & 17865 \\
\hline & Osbpl3 & 71720 & & Slc1a2 & 20511 & & Nfasc & 269116 \\
\hline & Esco2 & 71988 & & Steap2 & 74051 & miR-380-5p & Cdon & 57810 \\
\hline & 4930547N16Rik & 75317 & & Dcdc2a & 195208 & & Ccdc89 & 70054 \\
\hline \multirow[t]{12}{*}{ miR-669d-3p } & Cenpi & 102920 & miR-466 k & Zscan29 & 99334 & miR-410-3p & Pla2r1 & 18779 \\
\hline & Cysltr1 & 58861 & & Dcdc2a & 195208 & & Sema3e & 20349 \\
\hline & Gnai1 & 14677 & & Ptchd 1 & 211612 & miR-449a-5p & Gpr64 & 237175 \\
\hline & Zmat1 & 215693 & & Saa4 & 20211 & & H6pd & 100198 \\
\hline & Rgs4 & 19736 & miR-653-3p & |gf2bp1 & 140486 & miR-466n-3p & Mest & 17294 \\
\hline & Kif23 & 71819 & & Nfasc & 269116 & & Dcdc2a & 195208 \\
\hline & Fam55c & 385658 & & Ect2 & 13605 & $\mathrm{miR}-467 \mathrm{~g}$ & Cxcl5 & 20311 \\
\hline & Birc5 & 11799 & & Lox & 16948 & & Dcdc2a & 195208 \\
\hline & Aspm & 12316 & miR-669 h-3p & Snap25 & 20614 & miR-5113 & Gbp4 & 17472 \\
\hline & Bub1 & 12235 & & Steap2 & 74051 & & Slc7a2 & 11988 \\
\hline & Oip5 & 70645 & & Cysltr1 & 58861 & miR-670-3p & Evl & 14026 \\
\hline & Ckap2 & 80986 & & Rgs4 & 19736 & & $\mathrm{BCl} 2 \mid 15$ & 229672 \\
\hline \multirow[t]{7}{*}{ miR-98-3p } & Clspn & 269582 & miR-697 & Ckap4 & 216197 & miR-692 & Marcks & 17118 \\
\hline & Zfpm2 & 22762 & & Slc1a2 & 20511 & & Dcdc2a & 195208 \\
\hline & Nfasc & 269116 & & Fzd8 & 14370 & miR-693-3p & Akr1c14 & 105387 \\
\hline & Ect2 & 13605 & & Klf15 & 66277 & & Nfasc & 269116 \\
\hline & Ccna2 & 12428 & miR-881-5p & Serpine1 & 18787 & miR-701-3p & 1700029l01Rik & 70005 \\
\hline & Rad51 & 19361 & & Slc1a2 & 20511 & & Dcdc2a & 195208 \\
\hline & Dock11 & 75974 & & Steap2 & 74051 & miR-758-3p & Tpx2 & 72119 \\
\hline \multirow[t]{6}{*}{ miR-21-3p } & Nuf2 & 66977 & & Fmo5 & 14263 & & Zfpm2 & 22762 \\
\hline & Steap2 & 74051 & miR-9-5p & Fam132b & 227358 & miR-875-3p & $\mathrm{CxCl} 3$ & 330122 \\
\hline & Zfpm2 & 22762 & & Lhfp & 108927 & & Ehf & 13661 \\
\hline & Fgf13 & 14168 & & Galnt3 & 14425 & miR-122-5p & Samd5 & 320825 \\
\hline & Sema3e & 20349 & & Sort1 & 20661 & miR-134-5p & H6pd & 100198 \\
\hline & Top2a & 21973 & let-7f-2-3p & Gm13154 & 433804 & miR-182-3p & Lhfpl2 & 218454 \\
\hline \multirow[t]{6}{*}{ miR-30b-5p } & |gf2bp1 & 140486 & & Fam164a & 67306 & miR-188-5p & Rspo3 & 72780 \\
\hline & Slc1a2 & 20511 & & Ypel1 & 106369 & miR-1892 & Slc7a2 & 11988 \\
\hline & Cysltr1 & 58861 & miR-107-3p & Rttn & 246102 & miR-1897-5p & Marcks & 17118 \\
\hline & Gnai1 & 14677 & & Zfpm2 & 22762 & miR-193-3p & Abcc4 & 239273 \\
\hline & Lox & 16948 & & Shcbp1 & 20419 & miR-193-5p & Tspyl3 & 241732 \\
\hline & Nedd4l & 83814 & miR-124-5p & Klhl13 & 67455 & miR-1950 & $A x \mid$ & 26362 \\
\hline
\end{tabular}


Table 1 The microRNA targets regulated by SST (Continued)

\begin{tabular}{|c|c|c|c|c|c|c|c|c|}
\hline \multirow[t]{6}{*}{ miR-30d-5p } & Prr11 & 270906 & & Steap2 & 74051 & miR-1953 & Steap2 & 74051 \\
\hline & Cysltr1 & 58861 & & $\mathrm{Cd} 24 \mathrm{a}$ & 12484 & miR-195-3p & Cebpd & 12609 \\
\hline & Gnai1 & 14677 & miR-1947-3p & Prrx1 & 18933 & miR-200a-5p & Fgf13 & 14168 \\
\hline & Lox & 16948 & & Slc1a2 & 20511 & miR-200b-3p & Lhfp & 108927 \\
\hline & Rnf219 & 72486 & & Steap2 & 74051 & miR-203-5p & Abcc4 & 239273 \\
\hline & Nedd4I & 83814 & miR-200a-3p & Thbd & 21824 & miR-206-3p & Nedd9 & 18003 \\
\hline \multirow[t]{6}{*}{ miR-466a-5p } & Prc1 & 233406 & & Mbnl3 & 171170 & miR-214-3p & Slc7a2 & 11988 \\
\hline & Slcla2 & 20511 & & Lhfp & 108927 & miR-216b-3p & Akr1c14 & 105387 \\
\hline & Steap2 & 74051 & miR-291b-3p & Rtn1 & 104001 & miR-25-5p & Zfp365 & 216049 \\
\hline & Fam55c & 385658 & & Kif23 & 71819 & miR-298-3p & Ccdc89 & 70054 \\
\hline & Fgf23 & 64654 & & Kit & 16590 & miR-29c-3p & Pxdn & 69675 \\
\hline & Amotl1 & 75723 & miR-29b-2-5p & Fam55c & 385658 & miR-3062-5p & Ccdc89 & 70054 \\
\hline \multirow[t]{6}{*}{ miR-466o-3p } & Gtse1 & 29870 & & Gnai1 & 14677 & miR-3063-5p & Pak3 & 18481 \\
\hline & Zmat1 & 215693 & & Zmat1 & 215693 & miR-3064-5p & Fbln2 & 14115 \\
\hline & Kif23 & 71819 & miR-466i-3p & Tnfaip2 & 21928 & miR-3075-3p & Wisp1 & 22402 \\
\hline & Aspm & 12316 & & Slc7a2 & 11988 & miR-3085-3p & Abcc1 & 17250 \\
\hline & Gpr64 & 237175 & & Gbp4 & 17472 & miR-3094-5p & Fgf23 & 64654 \\
\hline & Serpinb1b & 282663 & miR-669c-3p & Tnfaip2 & 21928 & miR-3103-3p & Scarf2 & 224024 \\
\hline \multirow[t]{6}{*}{ miR-669 I-3p } & Bmf & 171543 & & Slc7a2 & 11988 & miR-3112-5p & Ptchd1 & 211612 \\
\hline & Fzd8 & 14370 & & Adm & 11535 & miR-322-5p & Fam164a & 67306 \\
\hline & Kit & 16590 & miR-669e-3p & Tia1 & 21841 & miR-326-5p & Aif1l & 108897 \\
\hline & Serpinb1b & 282663 & & Fgf13 & 14168 & miR-335-5p & Gclc & 14629 \\
\hline & Trim59 & 66949 & & Pak3 & 18481 & miR-3473d & B4galt6 & 56386 \\
\hline & Bmper & 73230 & miR-101a-3p & Sult4a1 & 29859 & miR-363-3p & Adm & 11535 \\
\hline \multirow[t]{5}{*}{ miR-30a-5p } & Prr11 & 270906 & & Mbnl3 & 171170 & $m i R-376 c-5 p$ & Prrx1 & 18933 \\
\hline & Cysltr1 & 58861 & miR-101a-5p & Klhl13 & 67455 & miR-378-3p & Sema3e & 20349 \\
\hline & Gnai1 & 14677 & & Mbnl3 & 171170 & miR-378b & Igf2bp3 & 140488 \\
\hline & Rnf219 & 72486 & miR-101b-3p & Sult4a1 & 29859 & miR-380-3p & Mbnl3 & 171170 \\
\hline & Nedd4l & 83814 & & Mbnl3 & 171170 & miR-382-3p & Sdpr & 20324 \\
\hline \multirow[t]{5}{*}{ miR-30e-5p } & Prr11 & 270906 & miR-105 & Ect2 & 13605 & miR-409-3p & Akr1c14 & 105387 \\
\hline & Cysltr1 & 58861 & & Zfpm2 & 22762 & miR-431-5p & Klf15 & 66277 \\
\hline & Gnai1 & 14677 & miR-142-5p & Depdc1a & 76131 & miR-463-5p & Pla2r1 & 18779 \\
\hline & Lox & 16948 & & Igf2bp3 & 140488 & miR-466i-5p & Dedc2a & 195208 \\
\hline & Rnf219 & 72486 & miR-181b-1-3p & Fgf13 & 14168 & miR-466 l-3p & Snhg11 & 319317 \\
\hline \multirow[t]{5}{*}{ miR-543-3p } & Mlf1 & 17349 & & Slc1a2 & 20511 & miR-470-5p & Steap4 & 117167 \\
\hline & Slc1a2 & 20511 & miR-1912-3p & Gpr137b & 83924 & miR-484 & Csf1 & 12977 \\
\hline & Cysltr1 & 58861 & & Ptchd1 & 211612 & miR-496-3p & Tspan8 & 216350 \\
\hline & Fut4 & 14345 & miR-1942 & Mxra8 & 74761 & miR-499-5p & Cdk1 & 12534 \\
\hline & Kifc2 & 16581 & & Zfpm2 & 22762 & miR-5101 & $\| 5$ ra & 16192 \\
\hline \multirow[t]{4}{*}{ let-7a-2-3p } & 4930486L24Rik & 214639 & miR-1a-1-5p & Ehf & 13661 & miR-5125 & Mllt11 & 56772 \\
\hline & Sema3e & 20349 & & Dcdc2a & 195208 & miR-5127 & Col4a5 & 12830 \\
\hline & $\mathrm{Cd} 24 \mathrm{a}$ & 12484 & miR-1b-5p & Ugt2b35 & 243085 & miR-5133 & Rasl12 & 70784 \\
\hline & Pamr1 & 210622 & & Tlr4 & 21898 & miR-544-3p & Snhg11 & 319317 \\
\hline \multirow[t]{2}{*}{ miR-137-3p } & Glis2 & 83396 & miR-219-5p & Tnfsf15 & 326623 & miR-675-3p & Mbnl3 & 171170 \\
\hline & Nfasc & 269116 & & Gprc5b & 64297 & miR-677-5p & Gclc & 14629 \\
\hline
\end{tabular}


Table 1 The microRNA targets regulated by SST (Continued)

\begin{tabular}{|c|c|c|c|c|c|c|c|c|}
\hline & Cep55 & 74107 & miR-26a-5p & Hpgd & 15446 & miR-712-5p & Cep55 & 74107 \\
\hline & Birc5 & 11799 & & Rgs4 & 19736 & miR-7a-5p & Mlph & 171531 \\
\hline \multirow[t]{4}{*}{ miR-149-5p } & B4galt6 & 56386 & miR-294-3p & Lass3 & 545975 & miR-877-3p & $\mathrm{Npr3}$ & 18162 \\
\hline & Pak3 & 18481 & & Zfpm2 & 22762 & miR-879-5p & $\mathrm{Hmmr}$ & 15366 \\
\hline & $\| 5$ ra & 16192 & miR-29a-3p & Col5a3 & 53867 & miR-881-3p & Ehf & 13661 \\
\hline & $A x \mid$ & 26362 & & Ppic & 19038 & & & \\
\hline \multirow[t]{4}{*}{ miR-194-5p } & Gas213 & 237436 & miR-3066-5p & Gpt2 & 108682 & & & \\
\hline & Fam164a & 67306 & & Ccna2 & 12428 & & & \\
\hline & Ppic & 19038 & miR-3071-5p & $\operatorname{lgf2bp} 1$ & 140486 & & & \\
\hline & Trim59 & 66949 & & Mbnl3 & 171170 & & & \\
\hline \multirow[t]{2}{*}{ miR-1964-5p } & Csdc2 & 105859 & miR-204-3p & Kirrel3 & 67703 & miR-465c-5p & Ugt2b1 & 71773 \\
\hline & Kirrel3 & 67703 & miR-23a-3p & Сур3а11 & 13112 & miR-466b-3p & Oas3 & 246727 \\
\hline let-7e-5p & Cyp2c50 & 107141 & miR-295-5p & Aldob & 230163 & miR-466f-3p & Npat & 244879 \\
\hline miR-126-5p & Ugt3a2 & 223337 & miR-30e-3p & Сур2f2 & 13107 & $m i R-466$ m-3p & Oas3 & 246727 \\
\hline miR-181a-5p & Nipal1 & 70701 & miR-328-5p & Cyp2d22 & 56448 & miR-5131 & Ccdc85b & 240514 \\
\hline miR-181b-5p & Nipal1 & 70701 & miR-344f-5p & Scd1 & 20249 & miR-551b-5p & 5033411D12Rik & 192136 \\
\hline miR-1960 & Mrc1 & 17533 & miR-3470a & Dnahc17 & 69926 & miR-676-5p & Slc27a5 & 26459 \\
\hline miR-19b-1-5p & Npat & 244879 & miR-465a-5p & Ugt2b1 & 71773 & miR-707 & Slcola1 & 28248 \\
\hline
\end{tabular}

"The MicroRNA name is obtained from the MicroRNA Database (miRDB version 4.0) website (http://mirdb.org) $[27,28]$.

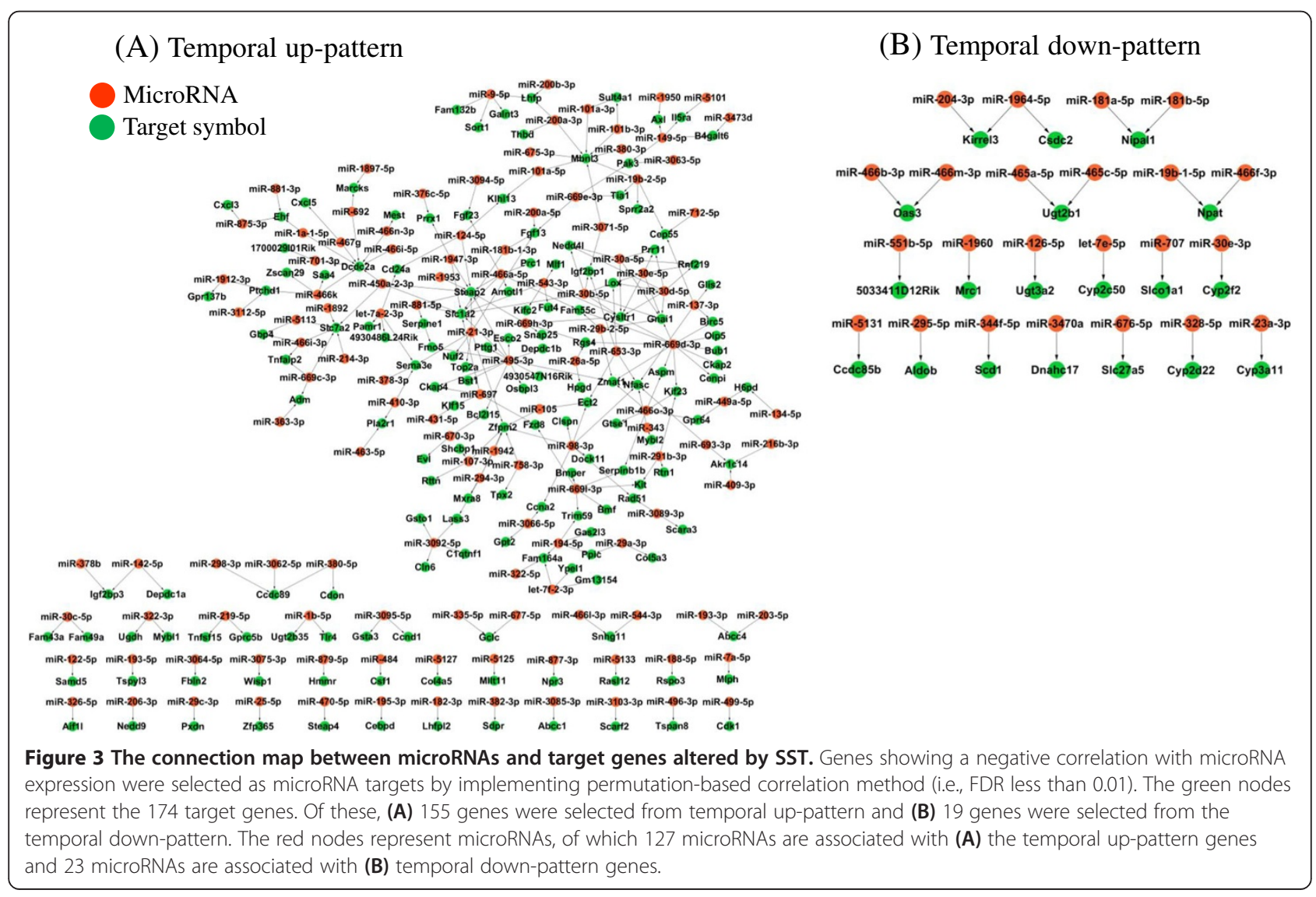


Table 2 Pathways enriched in temporal patterns by SST

\begin{tabular}{|c|c|c|c|c|c|c|c|}
\hline & & $\begin{array}{l}\text { Pathways from temporal } \\
\text { up-pattern (KEGG ID) }\end{array}$ & P-value* & $\mathrm{FDR}^{* *}$ & $\begin{array}{l}\text { Pathways from temporal down-pattern } \\
\text { (KEGG ID) }\end{array}$ & P-value & FDR \\
\hline \multirow[t]{17}{*}{ All genes } & \multirow{9}{*}{$\begin{array}{l}\text { Simple enrichment } \\
\text { analysis }\end{array}$} & \multirow[t]{11}{*}{ Cell cycle (mmu04110) } & \multirow[t]{9}{*}{$8.41 \mathrm{E}-05$} & \multirow[t]{9}{*}{$9.62 \mathrm{E}-03$} & Drug metabolism-cytochrome P450 (mmu00982) & $3.57 \mathrm{E}-10$ & 3.07E-08 \\
\hline & & & & & Systemic lupus erythematosus (mmu05322) & 7.97E-10 & 3.43E-08 \\
\hline & & & & & Complement and coagulation cascades (mmu04610) & $3.45 \mathrm{E}-08$ & $9.88 \mathrm{E}-07$ \\
\hline & & & & & Retinol metabolism (mmu00830) & $4.38 \mathrm{E}-08$ & $9.41 \mathrm{E}-07$ \\
\hline & & & & & $\begin{array}{l}\text { Metabolism of xenobiotics by cytochrome } \\
\text { P450 (mmu00980) }\end{array}$ & $1.15 \mathrm{E}-06$ & 1.97E-05 \\
\hline & & & & & Linoleic acid metabolism (mmu00591) & $2.04 \mathrm{E}-06$ & 2.92E-05 \\
\hline & & & & & Prion diseases (mmu05020) & $2.43 \mathrm{E}-05$ & 2.99E-04 \\
\hline & & & & & PPAR signaling pathway (mmu03320) & $3.42 \mathrm{E}-05$ & 3.67E-04 \\
\hline & & & & & Drug metabolism-other enzymes (mmu00983) & 4.87E-04 & 4.64E-03 \\
\hline & \multirow{8}{*}{$\begin{array}{c}\text { Topology-based } \\
\text { signaling pathway } \\
\text { analysis }\end{array}$} & & & & Systemic lupus erythematosus (mmu05322) & $1.74 \mathrm{E}-10$ & 1.36E-08 \\
\hline & & & & & $\begin{array}{l}\text { Complement and coagulation } \\
\text { cascades (mmu04610) }\end{array}$ & $4.55 \mathrm{E}-10$ & 1.77E-08 \\
\hline & & $\begin{array}{l}\text { Cytokine-cytokine receptor } \\
\text { interaction (mmu04060) }\end{array}$ & $1.69 \mathrm{E}-08$ & $1.84 \mathrm{E}-06$ & Prion diseases (mmu05020) & $2.82 \mathrm{E}-07$ & 7.33E-06 \\
\hline & & $\begin{array}{l}\text { Osteoclast differentiation } \\
\text { (mmu04380) }\end{array}$ & $4.88 \mathrm{E}-06$ & $2.66 \mathrm{E}-04$ & PPAR signaling pathway (mmu03320) & $1.42 \mathrm{E}-06$ & $2.78 \mathrm{E}-05$ \\
\hline & & Cell cycle (mmu04110) & $1.52 \mathrm{E}-04$ & $5.55 \mathrm{E}-03$ & Staphylococcus aureus infection (mmu05150) & $3.48 \mathrm{E}-06$ & 5.42E-05 \\
\hline & & & & & Serotonergic synapse (mmu04726) & $1.18 \mathrm{E}-05$ & 1.53E-04 \\
\hline & & & & & Alcoholism (mmu05034) & $2.48 \mathrm{E}-04$ & 2.77E-03 \\
\hline & & & & & $\begin{array}{l}\text { Endocrine and other factor-regulated } \\
\text { calcium reabsorption (mmu04961) }\end{array}$ & $7.76 \mathrm{E}-04$ & 7.57E-03 \\
\hline \multirow[t]{2}{*}{ MicroRNA targets } & $\begin{array}{l}\text { Simple enrichment } \\
\text { analysis }\end{array}$ & No pathway & & & $\begin{array}{l}\text { Metabolism of xenobiotics by cytochrome } \\
\text { P450 (mmu00980) }\end{array}$ & $1.45 \mathrm{E}-04$ & $3.19 \mathrm{E}-03$ \\
\hline & $\begin{array}{c}\text { Topology-based } \\
\text { signaling pathway } \\
\text { analysis }\end{array}$ & Cell cycle (mmu04110) & $5.46 \mathrm{E}-03$ & $1.00 \mathrm{E}-02$ & No pathway & & \\
\hline \multirow[t]{15}{*}{ Non-microRNA targets } & \multirow{7}{*}{$\begin{array}{l}\text { Simple enrichment } \\
\text { analysis }\end{array}$} & \multirow[t]{7}{*}{ No pathway } & & & Systemic lupus erythematosus (mmu05322) & $1.63 \mathrm{E}-10$ & $1.22 \mathrm{E}-08$ \\
\hline & & & & & Complement and coagulation cascades (mmu04610) & $9.24 \mathrm{E}-09$ & $3.46 \mathrm{E}-07$ \\
\hline & & & & & Drug metabolism (mmu00982) & $1.94 \mathrm{E}-07$ & 4.85E-06 \\
\hline & & & & & Prion diseases (mmu05020) & $1.20 \mathrm{E}-05$ & 2.26E-04 \\
\hline & & & & & Retinol metabolism (mmu00830) & $2.82 \mathrm{E}-05$ & 4.22E-04 \\
\hline & & & & & Linoleic acid metabolism (mmu00591) & $2.01 \mathrm{E}-04$ & $2.51 \mathrm{E}-03$ \\
\hline & & & & & PPAR signaling pathway (mmu03320) & $8.76 \mathrm{E}-04$ & 9.34E-03 \\
\hline & \multirow{8}{*}{$\begin{array}{c}\text { Topology-based } \\
\text { signaling pathway } \\
\text { analysis }\end{array}$} & \multirow{3}{*}{$\begin{array}{l}\text { Cytokine-cytokine receptor } \\
\text { interaction (mmu04060) }\end{array}$} & $1.63 \mathrm{E}-07$ & $1.42 \mathrm{E}-05$ & Systemic lupus erythematosus (mmu05322) & $3.95 \mathrm{E}-11$ & $3.00 \mathrm{E}-09$ \\
\hline & & & & & Complement and coagulation cascades (mmu04610) & $1.31 \mathrm{E}-10$ & 4.97E-09 \\
\hline & & & & & Prion diseases (mmu05020) & $1.32 \mathrm{E}-07$ & 3.34E-06 \\
\hline & & $\begin{array}{l}\text { NF-kappa B signaling } \\
\text { pathway (mmu04064) }\end{array}$ & 2.37E-05 & 8.95E-04 & Staphylococcus aureus infection (mmu05150) & $1.63 \mathrm{E}-06$ & 3.10E-05 \\
\hline & & $\begin{array}{l}\text { MAPK signaling } \\
\text { pathway (mmu04010) }\end{array}$ & $3.09 \mathrm{E}-05$ & 8.95E-04 & Serotonergic synapse (mmu04726) & $5.15 \mathrm{E}-05$ & 7.83E-04 \\
\hline & & \multirow{3}{*}{$\begin{array}{l}\text { Osteoclast differentiation } \\
\quad(\mathrm{mmu} 04380)\end{array}$} & \multirow[t]{3}{*}{ 4.17E-04 } & \multirow[t]{3}{*}{ 9.07E-03 } & PPAR signaling pathway (mmu03320) & $9.33 \mathrm{E}-05$ & $1.18 \mathrm{E}-03$ \\
\hline & & & & & $\begin{array}{l}\text { Endocrine and other factor-regulated } \\
\text { calcium reabsorption (mmu04961) }\end{array}$ & $6.65 \mathrm{E}-04$ & $7.22 \mathrm{E}-03$ \\
\hline & & & & & Alcoholism (mmu05034) & 9.07E-04 & 8.61E-03 \\
\hline
\end{tabular}

\footnotetext{
*For simple enrichment analysis, the $p$ values are calculated by the Fisher's exact test in the DAVID program [30]. For topology-based signaling pathway analysis, the $p$ value indicates the global pathway significance $p$ value $\left(\mathrm{P}_{\mathrm{G}}\right)$, which combines the enrichment $p$ values and the perturbation $p$ values in regard to pathway topology with a random bootstrap iteration number of 3000 [31].

** The false discovery rate (FDR) correction is measured by applying the Benjamini algorithm [30,31].
}

pathway analysis, which calculates the enrichment score by taking into account the topology of each signaling pathway, also showed that the cell cycle pathway (i.e., mmu04110) was significantly enriched from the temporal up-pattern, whereas diverse signaling pathways (e.g., immune-related pathways and metabolism-related path- 
ways) were selected as significant pathways from the temporal down-pattern. In addition, the cytokine-cytokine receptor interaction pathway (mmu04060) and the osteoclast differentiation pathway (mmu04380) were also significant pathways that were associated with the temporal up-pattern.

We measured temporal changes in pathway activity by using the expression levels of all genes in each pathway. Figure 4 shows that many diverse pathways were temporally activated or repressed, according to the SST treatment. Pathways enriched from the temporal up-pattern and down-pattern showed temporally increased and decreased activity, respectively.

The functional association of microRNA target genes shows that only one pathway-the cell cycle pathway (mmu04110) - was statistically significant from the temporal up-pattern (the FDR was less than 0.01). From the temporal down-pattern, we measured one pathway that was also statistically significant: the xenobiotics metabolism pathway (mmu00980). On the other hand, nonmicroRNA targets from the temporal up-pattern were associated with signaling pathways such as the cytokinecytokine receptor interaction pathway (mmu04060), the NF- $\kappa B$ signaling pathway (mmu04064), the mitogenactivated protein kinase (MAPK) pathway (mmu04010), and the osteoclast differentiation pathway (mmu04380). However, non-microRNA targets from the temporal down-pattern were associated with diverse pathways such as immune-related pathways and metabolismrelated pathways. (see Additional file 1: Figure S3) shows the positions of the temporally regulated genes in each significant pathway.

By comparing pathways involved in the microRNA targets and microRNA non-targets, we speculated that microRNA was specific for the regulation of the cell cycle pathway from temporal up-pattern and the xenobiotics metabolism pathway from the temporal down-pattern.

\section{Integration of multiple pathways}

We found that only a few pathways (e.g., cell cycle pathway and xenobiotics metabolism pathway) were associated with microRNA target genes regulated by SST. However, as an individual gene, the microRNA target could play critical roles in diverse pathways. Therefore, we integrated all pathways that were significantly enriched by SST to identify key microRNA targets. From multiple pathways associated with the temporal up-pattern, the core microRNA targets selected were CCNA2, PTTG1, CDK1, CCNB2, CDC25B, CCL7, MAPK12 and ESPL1 (Figure 5A). From the temporal down-pattern, CYP2F2, CYP3A11, and CYP2C50 were selected as nodes with multiple roles (Figure 5B). The pathways containing these core targets of microRNA are shown below each network structure.

\section{TFBS analysis}

The functional segregation of genes, based on the expression pattern, suggests that the gene transcription process would be the direct regulatory target of SST. Therefore, we investigated the possible association of the TFBS structure on the gene expression after SST treatment. By using the promoter region $(-2000 \mathrm{bp}$ to $+500 \mathrm{bp}$ from the transcription start site) of genes included in the temporal patterns, the correlation matrix of genes based on TFBS similarity was measured. The resultant clustering profile shows that genes in the temporal up-pattern are clearly distinguished from genes in the temporal down-pattern. As Figure 6A shows, two subgroups of genes were tightly clustered (i.e., Upcluster and Down-cluster), which were primarily composed of genes from the temporal up-pattern and down-pattern, respectively. In addition to the main subgroups, there were other subgroups that also consisted exclusively of temporal up-pattern or down-pattern genes. The putative target genes of the microRNAs were interestingly also clustered into small subgroups (Figure 6A). This segregation of microRNA targets was more clearly observed in the temporal up-pattern genes (Figure 6B). One subgroup of microRNA targets was closely correlated with the similar TFBS structure (depicted as MicroRNA cluster in Figure 6B). MicroRNA target genes from the temporal down-pattern were also primarily concentrated on one cluster, although the number of target genes was small (Figure 6C). This separation of genes based on TFBS similarity indicates the presence of common cis-elements in the SST-regulated gene expression.

\section{Discussion}

Despite the clinical usefulness of traditional herbal medicine, the complex nature of herbal chemical components prevents the elucidation of their exact molecular mechanisms. The herbal preparation of SST is also widely prescribed for the treatment of diverse liver diseases, but without clear understanding of its molecular mechanism [1]. What further complicates the situation is that SST is composed of seven different herbal plants (see Additional file 1: Table S1). Therefore, understanding the molecular activity of SST is limited when focusing on only a few major components or certain kinds of genes.

In the present study, we measured the global changes of genes and microRNAs expression induced by SST in cultured primary mouse hepatocytes, because the liver is a primary target organ of SST and is responsible for metabolizing xenobiotics. The expression profile shows two temporal expression patterns of genes after SST treatment, but no clear temporal pattern in microRNA expression (Figure 2). The microRNA expression levels after SST treatment were lower than the expression levels of genes. This suggests that a small number of 




Figure 4 (See legend on next page.) 
(See figure on previous page.)

Figure 4 Pathway activities altered by SST in primary mouse hepatocytes. The temporal change of pathway activity is measured by linearly combining the logarithmic expression value of all genes in each pathway and then clustering them hierarchically. The columns represent individual samples and the rows represent the activity of the pathways. Red indicates high activity and green indicates low activity, as indicated by a scale bar with arbitrary units. The pathways selected as significant in enrichment analysis are indicated in red for temporal up-pattern and blue for temporal down-pattern.

microRNAs can regulate many genes. Therefore, it is critical to identify accurately the microRNA target genes. We used a correlation-based permutation approach to exclude possible false-positive links between microRNA and its putative target gene expression. The resultant 174 microRNA target genes were obtained from 463 temporal up-pattern genes and 19 targets were obtained from 177 temporal down-pattern genes (Figure 3 and Table 1). This indicates that microRNA is especially concentrated in the regulation of temporal up-pattern genes ( $p$ value $<0.001)$.
In addition to this unbalanced distribution of microRNA target genes, different biological functions were associated with microRNA targets in the two temporal patterns. For example, cell cycle pathway (mmu04110) was specifically involved in microRNA targets from the temporal up-pattern genes. On the other hand, nonmicroRNA target genes from temporal up-pattern genes were significantly associated (the FDR was less than 0.01) with cell signaling pathways such as the cytokinecytokine receptor interaction pathway (mmu04060), the NF- $\mathrm{BB}$ signaling pathway (mmu04064), the MAPK
(A)
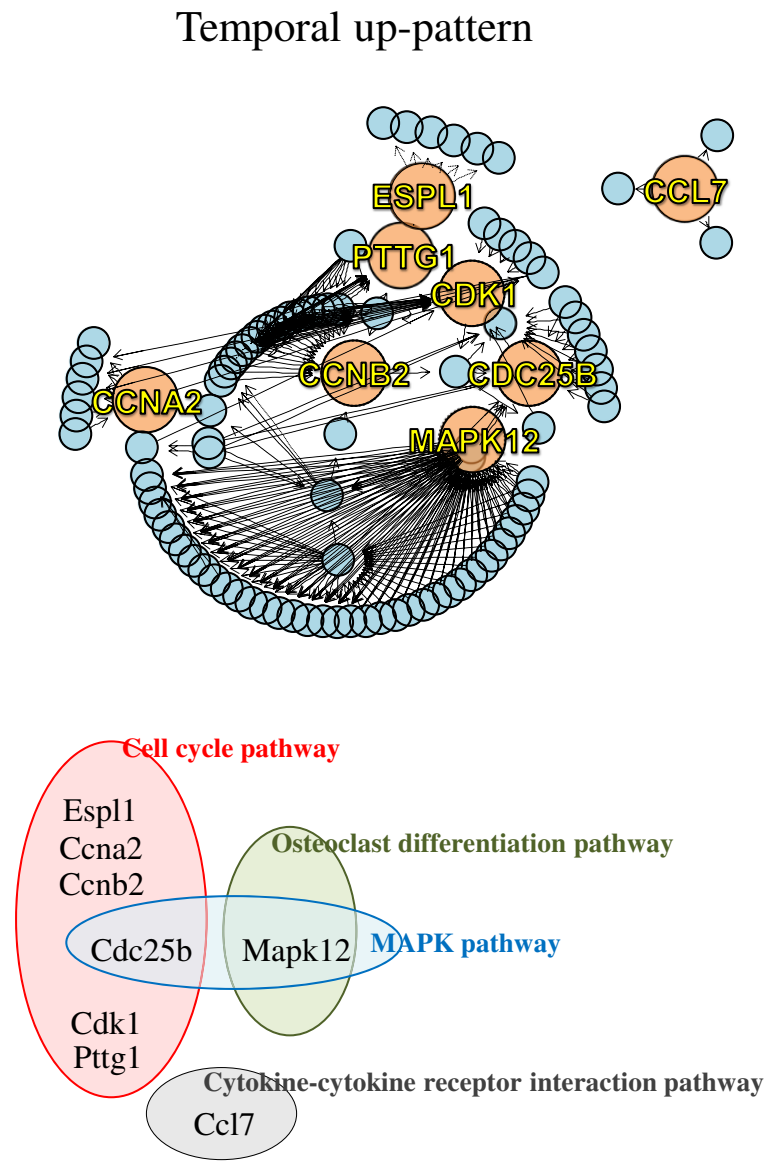

(B)

\section{Temporal down-pattern}
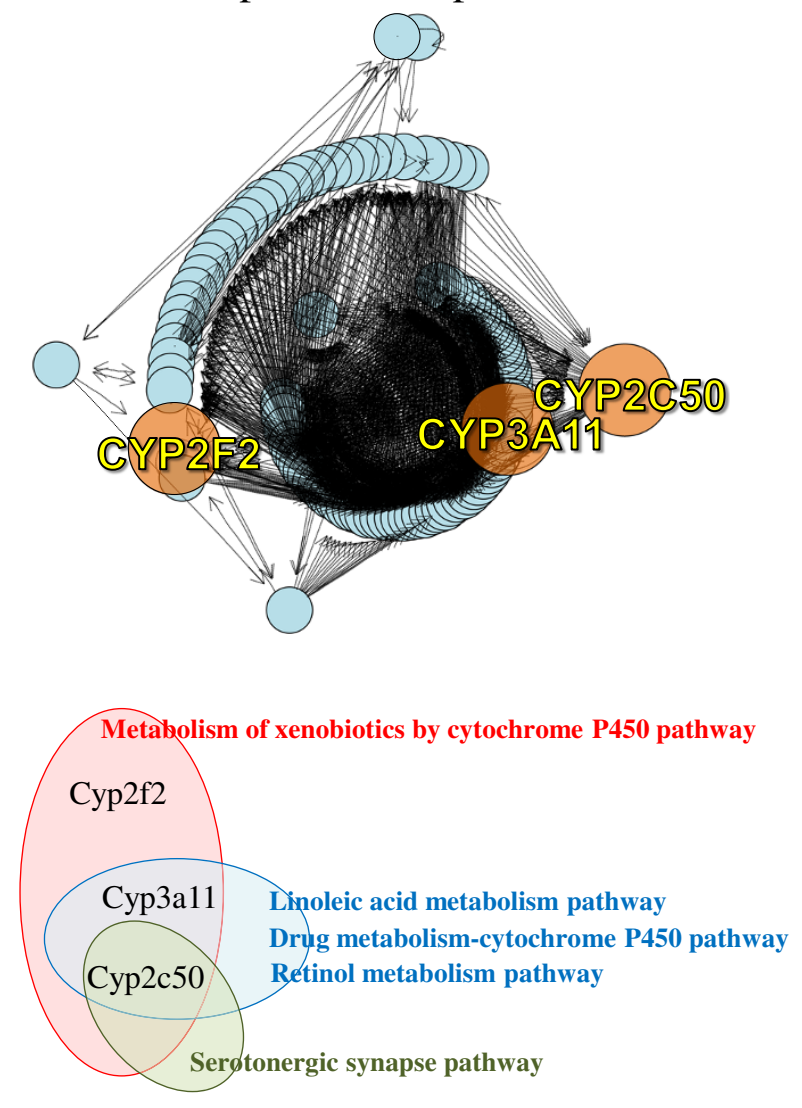

Figure 5 The core microRNA target genes in multiple pathways regulated by SST. (A) Nodes with high relative betweenness centrality were selected as the core microRNA targets in multiple pathways enriched in (A) the temporal up-pattern genes and (B) the temporal down-pattern genes. Each circle represents an individual gene node and each arrow represents its regulatory edge type. Out-going edges reflect nodes that act as regulators, whereas in-going edges reflect nodes that are subject to intermolecular regulations. The orange circles indicate the core nodes with a relative betweenness centrality greater than 0.01 . The pathways, including core nodes genes, are also indicated schematically. 


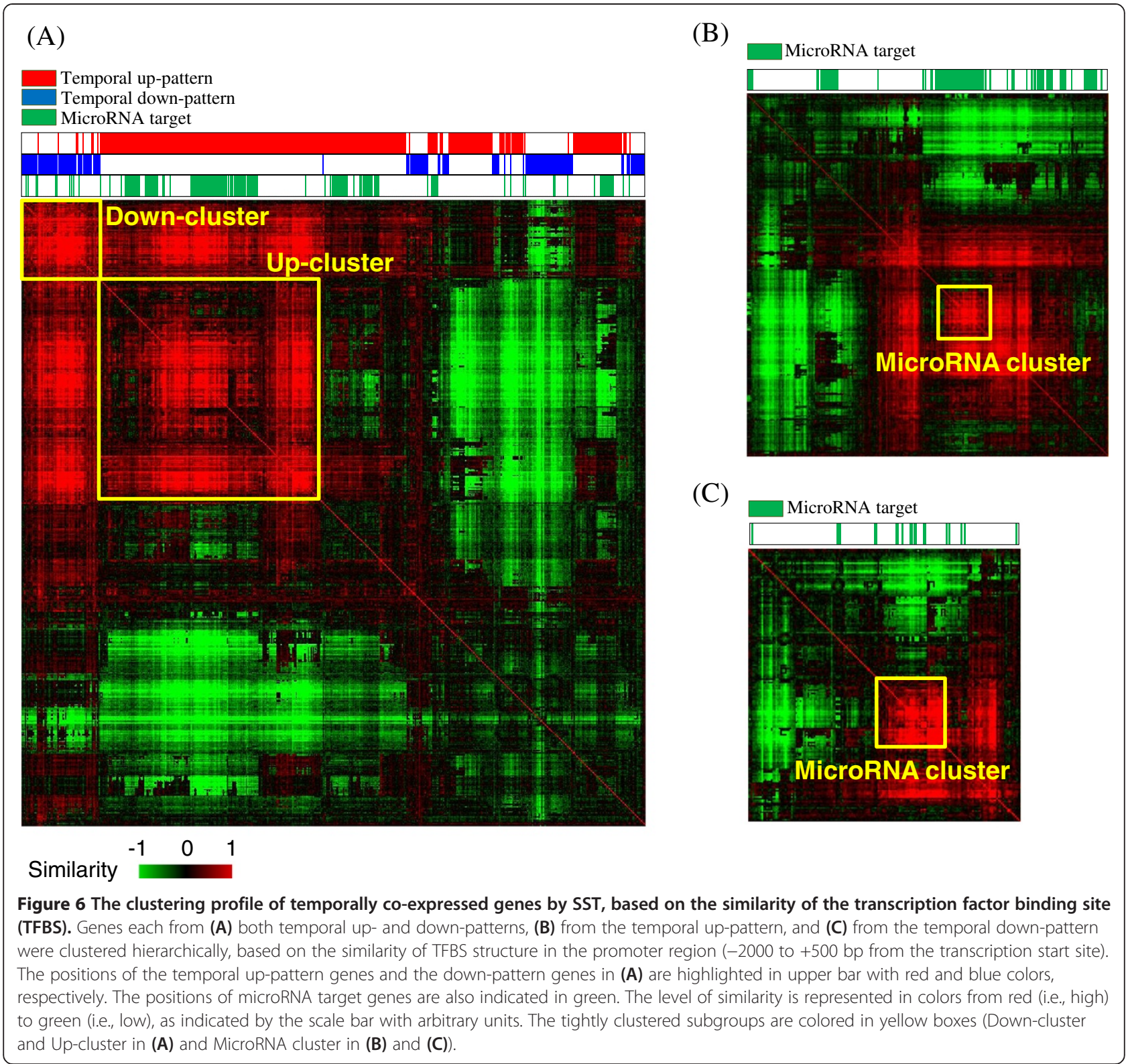

signaling pathway (mmu04010), and the osteoclast differentiation pathway (mmu04380) (Table 2). Unlike the temporal up-pattern, microRNA targets from the temporal down-pattern were associated only with the xenobiotics metabolism pathway (mmu00980). Non-microRNA targets from the temporal down-pattern were involved in diverse pathways, among which were two primary categories: the immune-related pathway and the metabolism-related pathway. However, the number of microRNA targets from the temporal down-pattern was small. The SST-enriched peroxisome proliferator-activated receptor (PPAR) pathway is critical in regulating metabolism and proliferation by modulating E2F and AKT signaling in the liver regeneration process [39].
The temporal change of activity plot (Figure 4) indicated that many other pathways in addition to pathways listed in Table 2 were also activated or suppressed, reflecting the fact that diverse biological functions were influenced by the SST treatment. As expected, the cell cycle pathway (mmu04110) from the temporal uppattern showed increased activity, whereas the immunerelated pathways and drug metabolism pathways from the temporal down-pattern showed decreased activity. The regulatory role of SST on cell proliferation has interestingly been previously reported in studies indicating that SST has an antiproliferative effect on hepatocarcinoma primarily because of anticarcinogenic components such as baicalein, baicalin, and saikosaponin $[2,40]$. However, 
clinical evidence and recent reports also suggest that SST enhances liver function by promoting the regeneration of the liver in animal models [6,7]. Therefore, activation of cell cycle pathway and MAPK pathway in the present study could be explained by this liver-regenerative effect of SST.

Another major clinical effect of SST is immuno-modulatory activity in diverse diseases [41,42]. As evidenced in previous reports, SST can activate or repress immune processes, depending on the cell type and the clinical situation $[9,43]$. In our results, SST activated immune pathways such as the cytokine receptor pathway (mmu04060), the TNF signaling pathway (mmu04668), rheumatoid arthritis pathway (mmu05323), NOD-like receptor signaling pathway (mmu04621) but it also repressed other immune-related pathways such as the systemic lupus erythematosus pathway (mmu05322), the complement and coagulation pathway (mmu04610), and the Staphylococcus aureus infection pathway (mmu05150) (Figure 4).

This coordinated change, induced by SST on the activity of multiple pathways, implicates a common regulatory mechanism controlling the multiple pathways. We interestingly observed that some microRNA targets (e.g., CCNA2, PTTG1, CDK1, CCNB2, CDC25B, CCL7, MAPK12, and ESPL1 from the temporal up-pattern genes and CYP2F2, CYP3A11, and CYP2C50 from the temporal down-pattern genes) can act as core targets connected with multiple significant pathways from nonmicroRNA targets (Figure 5)

We mentioned in the previous paragraph that signal pathways regulated by SST (e.g., the cell cycle pathway, PPAR pathway, and MAPK pathway) could be associated with the liver regenerative activity of SST. This can be also confirmed by using individual core node genes. For example, CCNA2 and CCL7, main elements of cell cycle pathways and the cytokine receptor pathway, respectively, are associated with liver regeneration in the rat liver $[44,45]$. Also CDC25B can regulate mouse liver regeneration in association with FOXM1 by promoting hepatocyte proliferation [46,47]. CDK1, another key element in the cell cycle pathway, plays an essential role in the control of DNA replication in liver regeneration [48]. These previous reports suggest that core microRNA target genes in temporal up-pattern could be associated with the liver regeneration function of SST by enhancing cell proliferation function. On the other hand, core microRNA target genes in the temporal downpattern (e.g., CYP2F2, CYP3A11, and CYP2C50) are exclusively associated with cytochrome P450 metabolism. However, there is interesting evidence that genes included in the cytochrome P450 family are also associated with liver regeneration. For example, early reduction of CYP activity has been observed in the regenerating rat liver, although the exact mechanism has not been elucidated [49]. The transcription of cytochrome P450 genes, including CYP3A11, moreover is reportedly suppressed by immune responses such as TNF- $\alpha$ in primary hepatocytes and hepatoma cells [50-52]. In consistent with the findings of previous reports, we observed the downregulation of cytochrome $\mathrm{P} 450$ metabolism pathways and the activation of the cytokine pathway (mmu04060) and TNF signaling pathway (mmu04668) by SST (Table 2 and Figure 4), which imply the involvement of drug metabolism pathway and immune-pathways on liver regeneration process. To conclude, pathways identified in present study such as cell cycle pathway, drug metabolism-cytochrome P450 pathway and immune-related pathways, and individual core node genes could be possible molecular targets involved in liver regenerative process induced by SST. However, considering that SST has diverse pharmacological activities on various pathological conditions, the roles of these pathways and core node genes should be more precisely measured in a variety of physiological models.

We also observed that this coordinated regulation of gene expression by SST was predisposed in the genomic structure. As Figure 6A shows, the similarity in measurements of the TFBS clearly distinguished temporal up-pattern genes from temporal down-pattern genes. The present results imply that common cis-elements present in the promoter region of the genes could determine the temporal co-expression of genes induced by SST. Moreover, considering functions associated with each temporal pattern, the difference in TFBS structure between the two temporal patterns may be related to biological functions associated with each temporal pattern. For a clearer conclusion, a TFBS analysis should be performed of all genes at a genome level. It should also be elucidated whether resultant genes with a similar TFBS structure may be co-expressed by SST. What was more intriguing was that putative microRNA target genes also were clustered into separate subgroups, especially in the temporal up-pattern genes (Figure 6B). Recent research reveals that microRNA is involved in the promoter methylation of target genes to regulate the transcription level in association with transcription factors [53] and that this mechanism of gene expression would form the global regulatory network [12,54-56]; however, we do not know whether methylation-based regulation by microRNA is also involved in the present study. Moreover, there is no report on the role of the TFBS structure on the regulation of gene expression by microRNA. Therefore, we expect that our finding could give an important clue about the novel mechanism of gene expression by microRNA.

\section{Conclusions}

The present study is the first to indicate that SST systematically regulates gene expression by microRNA. We 
demonstrated that temporally up-regulated pattern by SST was associated with signaling pathways, including the cell cycle pathway, whereas the temporally downregulated pattern included drug metabolism-related pathways and immune-related pathways, all of which could possibly contribute to the liver regenerative activity of SST. Also, this complex gene expression demonstrates that the effects of SST would be exerted from a delicately regulated mechanism on a genome-wide scale.

\section{Additional file}

Additional file 1: Table S1. Constituents of sho-saiko-to (SST). Table S2. List of genes in temporal patterns. Figure S1. The cytotoxic effect of Sho-saiko-to (SST) on primary hepatocytes. Hepatocytes are first cultured in 48-well plates at a density of $1.0 \times 105$ cells/well for 24 hours. After incubation, the cells are washed with phosphate-buffered saline and treated with different concentrations of SST $(0.1-1.0 \mathrm{mg} / \mathrm{mL})$ for 24 hours. Viability is measured in triplicate by using an in vitro colorimetric method (i.e., methyl thiazolyl tetrazoliym [MTT] assay). The viability is presented as the mean standard deviation (S.D.). Figure S2. Quantitative real-time polymerase chain reaction (Q-PCR). Mouse primary hepatocytes are treated with $500 \mathrm{\mu g} / \mathrm{mL}$ of SST at a density of $1.0 \times 106$ cells/60 mm dish for 1-24 hours in triplicate. The mRNA and microRNA are then reversetranscribed amplified, and detected by using Tagman probes (ABI, USA). The Q-PCR results are presented as the mean standard deviation (S.D.). Figure S3. Pathways enriched in the temporal up-pattern and temporal down-pattern. The position of each gene is denoted by red for the temporal up-pattern or blue for the temporal down-pattern in the pathways.

\section{Abbreviations}

SST: Sho-saiko-to; HBSS: Hank's balanced salt solution; STEM: Short time-series expression miner; FDR: False discovery rate; SPIA: Signaling pathway impact analysis; KEGG: Kyoto encyclopedia of genes and genomes; TFBS: Transcription factor binding site; UniPROBE: Universal PBM Resource for OligonucleotideBinding Evaluation; MAPK: Mitogen-activated protein kinase.

\section{Competing interests}

The authors declare that they have no competing interests.

\section{Authors' contributions}

KHS and YHK: conception, design of the experiment and preparation of the manuscript. BYK: conception, design of the experiment, analysis of data and preparation of the manuscript. All authors have read and approved the final manuscript.

\section{Acknowledgements}

The authors would like to thank Dr. Hyeun Kyoo Shin (Basic Herbal Medicine Research Group, Korea Institute of Oriental Medicine, Republic of Korea) for supporting SST. This research was supported by the "study of high frequency TKM prescription based on microRNA (C13020)" funded by SME Partnership Center of Korea Institute of Oriental Medicine (KIOM) and in part by a grant (KIOM-2010-2) from the Inter-Institutional Collaboration Research Program under the Korea Research Council of Fundamental Science \& Technology (KRCF).

\section{Author details}

'SME Partnership Center, Korea Institute of Oriental Medicine, Daejeon 305-811, Republic of Korea. ${ }^{2}$ Herbal Medicine Research Division, Korea Institute of Oriental Medicine, 1672 Yuseongdae-ro, Yuseong-gu, Daejeon 305-811, Republic of Korea. ${ }^{3}$ University of Science and Technology, 217 Gajeong-ro, Yuseong-gu, Daejeon 305-333, Republic of Korea.

Received: 16 September 2013 Accepted: 31 December 2013 Published: 11 January 2014

\section{References}

1. Ohtake N, Nakai Y, Yamamoto M, Sakakibara I, Takeda S, Amagaya S, Aburada MJ, Chromatogr B: Separation and isolation methods for analysis of the active principles of Sho-saiko-to (SST) oriental medicine. Analyt Technol Biomed Life Sci 2004, 812:135-148.

2. Shimizu I: Sho-saiko-to: Japanese herbal medicine for protection against hepatic fibrosis and carcinoma. J Gastroenterol Hepatol 2000, 15(Suppl):D84-D90.

3. Morgan TR: Chemoprevention of hepatocellular carcinoma in chronic hepatitis C. Recent Results Cancer Res 2011, 188:85-99.

4. Shiota G, Maeta Y, Mukoyama T, Yanagidani A, Udagawa A, Oyama K, Yashima K, Kishimoto Y, Nakai Y, Miura T, Ito H, Murawaki Y, Kawasaki H: Effects of Sho-Saiko-to on hepatocarcinogenesis and 8-hydroxy-2'-deoxyguanosine formation. Hepatology 2002, 35:1125-1133.

5. Chen MH, Chen JC, Tsai CC, Wang WC, Chang DC, Lin CC, Hsieh HY: Sho-saiko-to prevents liver fibrosis induced by bile duct ligation in rats. Am J Chin Med 2004, 32:195-207.

6. Miyamura M, Ono M, Kyotani S, Nishioka Y: Effects of sho-saiko-to extract on fibrosis and regeneration of the liver in rats. J Pharm Pharmacol 1998, 50:97-105.

7. Nakata R, Tsukamoto I, Miyoshi M, Kojo S: Liver regeneration after carbon tetrachloride intoxication in the rat. Biochem Pharmacol 1985, 34:586-588.

8. Ohtake N, Yamamoto M, Takeda S, Aburada M, Ishige A, Watanabe K, Inoue M: The herbal medicine Sho-saiko-to selectively inhibits CD8+ T-cell proliferation. Eur J Pharmacol 2005, 507:301-310.

9. Chen MH, Chen JC, Tsai CC, Wang WC, Chang DC, Tu DG, Hsieh HY: The role of TGF-beta 1 and cytokines in the modulation of liver fibrosis by Sho-saiko-to in rat's bile duct ligated model. J Ethnopharmacol 2005, 97:7-13.

10. Sanoudou D, Mountzios G, Arvanitis DA, Pectasides D: Array-based pharmacogenomics of molecular-targeted therapies in oncology. Pharmacogenomics J 2012, 12:185-196.

11. Rukov $\mathrm{J}$, Shomron N: MicroRNA pharmacogenomics: post-transcriptional regulation of drug response. Trends Mol Med 2011, 17:412-423.

12. Chen K, Rajewsky N: The evolution of gene regulation by transcription factors and microRNAs. Nat Rev Genet 2007, 8:93-103.

13. Friedman RC, Farh KK, Burge CB, Bartel DP: Most mammalian mRNAs are conserved targets of microRNAs. Genome Res 2009, 19:92-105.

14. Creighton CJ, Hernandez-Herrera A, Jacobsen A, Levine DA, Mankoo P, Schultz N, Du Y, Zhang Y, Larsson E, Sheridan R, Xiao W, Spellman PT, Getz G, Wheeler DA, Perou CM, Gibbs RA, Sander C, Hayes DN, Gunaratne PH: Integrated analyses of microRNAs demonstrate their widespread influence on gene expression in high-grade serous ovarian carcinoma. Cancer genome atlas research network. PLoS One 2012, 7:e34546.

15. Genovesi LA, Carter KW, Gottardo NG, Giles KM, Dallas PB: Integrated analysis of miRNA and mRNA expression in childhood medulloblastoma compared with neural stem cells. PLoS One 2011, 6:e23935.

16. Cho JH, Gelinas R, Wang K, Etheridge A, Piper MG, Batte K, Dakhallah D, Price J, Bornman D, Zhang S, Marsh C, Galas D: Systems biology of interstitial lung diseases: integration of mRNA and microRNA expression changes. BMC Med Genomics 2011, 4:8.

17. Su Z, Xia J, Zhao Z: Functional complementation between transcriptional methylation regulation and post-transcriptional microRNA regulation in the human genome. BMC Genomics 2011, 12(Suppl 5):S15.

18. Wiench B, Chen YR, Paulsen M, Hamm R, Schröder S, Yang NS, Efferth T: Integration of different "-omics" technologies identifies inhibition of the IGF1R-Akt-mTOR signaling cascade involved in the cytotoxic effect of shikonin against leukemia cells. Evid Based Complement Alternat Med 2013, 2013:818709.

19. Wen Z, Wang Z, Wang S, Ravula R, Yang L, Xu J, Wang C, Zuo Z, Chow MS, Shi $L$, Huang $Y$ : Discovery of molecular mechanisms of traditional Chinese medicinal formula Si-Wu-Tang using gene expression microarray and connectivity map. PLoS One 2011, 6:e18278.

20. Kim BY, Lee J, Park SJ, Bang OS, Kim NS: Gene expression profile of the A549 human Non-small cell lung carcinoma cell line following treatment with the seeds of descurainia Sophia, a potential anticancer drug. Evid Based Complement Alternat Med 2013, 2013:584604.

21. Kim BY, Cao LH, Kim JY: Common responses in gene expression by Ephedra herba in brain and heart of mouse. Phytother Res 2011, 25:1440-1446.

22. Shin IS, Lee MY, Kim Y, Seo CS, Kim JH, Shin HK: Subacute toxicity and stability of Soshiho-tang, a traditional herbal formula, in Sprague-Dawley rats. BMC Complement Altern Med 2012, 12:266. 
23. Park H, Song KH, Jung PM, Kim JE, Ro H, Kim MY, Ma JY: Inhibitory effect of arctigenin from fructus arctii extract on melanin synthesis via repression of tyrosinase expression. Evid Based Complement Alternat Med 2013, 2013:965312.

24. Song KH, Li T, Owsley E, Chiang JY: A putative role of micro RNA in regulation of cholesterol 7alpha-hydroxylase expression in human hepatocytes. J Lipid Res 2010, 51:2223-2233.

25. Bolstad BM, Irizarry RA, Astrand M, Speed TP: A comparison of normalization methods for high density oligonucleotide array data based on variance and bias. Bioinformatics 2003, 19:185-193.

26. Ernst J, Bar-Joseph Z: STEM: a tool for the analysis of short time series gene expression data. BMC Bioinforma 2006, 7:191

27. Wang $X$, el Naqa IM: Prediction of both conserved and nonconserved microRNA targets in animals. Bioinformatics 2008, 24:325-332.

28. Wang $X$ : miRDB: a microRNA target prediction and functional annotation database with a wiki interface. RNA 2008, 14:1012-1017.

29. Lee SY, Song KH, Koo I, Lee KH, Suh KS, Kim BY: Comparison of pathways associated with hepatitis B- and C-infected hepatocellular carcinoma using pathway-based class discrimination method. Genomics 2012, 99:347-354.

30. Dennis G Jr, Sherman BT, Hosack DA, Yang J, Gao W, Lane HC, Lempicki RA: DAVID: Database for Annotation, Visualization, and Integrated Discovery. Genome Biol 2003, 4:P3.

31. Tarca AL, Draghici S, Khatri P, Hassan SS, Mittal P, Kim JS, Kim CJ, Kusanovic JP, Romero R: A novel signaling pathway impact analysis. Bioinformatics 2009, 25:75-82.

32. Bang J, Jeon WK, Lee IS, Han JS, Kim BY: Biphasic functional regulation in hippocampus of Rat with chronic cerebral hypoperfusion induced by permanent occlusion of bilateral common carotid artery. PLoS One 2013, 8:e70093.

33. Zhang JD, Wiemann S: KEGGgraph: a graph approach to KEGG PATHWAY in R and bioconductor. Bioinformatics 2009, 25:1470-1471.

34. Shannon P: MotifDb: an annotated collection of protein-DNA binding sequence motifs. R package version 1.2.2. [http://www.bioconductor.org/ packages//2.11/bioc/html/MotifDb.html]

35. Portales-Casamar E, Thongjuea S, Kwon AT, Arenillas D, Zhao X, Valen E, Yusuf D, Lenhard B, Wasserman WW, Sandelin A: JASPAR 2010: the greatly expanded open-access database of transcription factor binding profiles. Nucleic Acids Res 2010, 38:D105-D110.

36. Wasserman WW, Sandelin A: Applied bioinformatics for the identification of regulatory elements. Nat Rev Genet 2004, 5:276-587.

37. Newburger DE, Bulyk ML: UniPROBE: an online database of protein binding microarray data on protein-DNA interactions. Nucleic Acids Res 2009, 37:D77-D82

38. Veerla S, Hoglund M: Analysis of promoter regions of coexpressed genes identified by microarray analysis. BMC Bioinforma 2006, 7:384.

39. Liu HX, Fang Y, Hu Y, Gonzalez FJ, Fang J, Wan YJ: PPARß Regulates Liver Regeneration by Modulating Akt and E2f Signaling. PLoS One 2013, 8:e65644.

40. Inoue T, Jackson EK: Strong antiproliferative effects of baicalein in cultured rat hepatic stellate cells. Eur J Pharmacol 1999, 378:129-135.

41. Borchers AT, Sakai S, Henderson GL, Harkey MR, Keen CL, Stern JS, Terasawa K, Gershwin ME: Shosaiko-to and other Kampo (Japanese herbal) medicines: a review of their immunomodulatory activities. $J$ Ethnopharmacol 2000, 73:1-13.

42. Ohtake $N$, Nakai $Y$, Yamamoto $M$, Ishige A, Sasaki H, Fukuda $K$, Hayashi S, Hayakawa S: The herbal medicine Shosaiko-to exerts different modulating effects on lung local immune responses among mouse strains. Int Immunopharmacol 2002, 2:357-366.

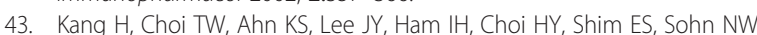
Upregulation of interferon-gamma and interleukin-4, Th cell-derived cytokines by So-Shi-Ho-Tang (Sho-Saiko-To) occurs at the level of antigen presenting cells, but not CD4 T cells. J Ethnopharmacol 2009, 123:6-14.

44. Wang WB, Fan JM, Zhang XL, Xu J, Yao W: Serial expression analysis of liver regeneration-related genes in rat regenerating liver. Mol Biotechnol 2009, 43:221-231.

45. Chen X, Xu C, Zhang F, Ma J: Comparative analysis of expression profiles of chemokines, chemokine receptors, and components of signaling pathways mediated by chemokines in eight cell types during rat liver regeneration. Genome 2010, 53:608-618.

46. Wang X, Kiyokawa H, Dennewitz MB, Costa RH: The Forkhead Box m1b transcription factor is essential for hepatocyte DNA replication and mitosis during mouse liver regeneration. Proc Natl Acad Sci U S A 2002, 99:16881-16886
47. Wang X, Krupczak-Hollis K, Tan Y, Dennewitz MB, Adami GR, Costa RH: Increased hepatic Forkhead Box M1B (FoxM1B) levels in old-aged mice stimulated liver regeneration through diminished p27Kip1 protein levels and increased Cdc25B expression. J Biol Chem 2002, 277:44310-44316.

48. Garnier D, Loyer P, Ribault C, Guguen-Guillouzo C, Corlu A: Cyclin-dependent kinase 1 plays a critical role in DNA replication control during rat liver regeneration. Hepatology 2009, 50:1946-1956

49. Starkel $P$, Laurent $S$, Petit $M$, van den Berge $V$, Lambotte $L$, Horsmans $Y$ : Early down-regulation of cytochrome P450 3A and 2E1 in the regenerating rat liver is not related to the loss of liver mass or the process of cellular proliferation. Liver 2000, 20:405-410.

50. Morgan ET, Goralski KB, Piquette-Miller M, Renton KW, Robertson GR, Chaluvadi MR, Charles KA, Clarke SJ, Kacevska M, Liddle C, Richardson TA, Sharma R, Sinal $\mathrm{CJ}$ : Regulation of drug-metabolizing enzymes and transporters in infection, inflammation, and cancer. Drug Metab Dispos 2008, 36:205-216.

51. Aitken AE, Richardson TA, Morgan ET: Regulation of drug-metabolizing enzymes and transporters in inflammation. Annu Rev Pharmacol Toxicol 2006, 46:123-149.

52. Morgan ET: Regulation of cytochromes P450 during inflammation and infection. Drug Metab Rev 1997, 29:1129-1188.

53. Taguchi $\mathrm{YH}$ : MicroRNA-mediated regulation of target genes in several brain regions is correlated to both microRNA-targeting-specific promoter methylation and differential microRNA expression. Bio Data Min 2013, 6:11.

54. Shalgi R, Lieber D, Oren M, Pilpel Y: Global and local architecture of the mammalian microRNA-transcription factor regulatory network. PLOS Comput Biol 2007, 6:e131.

55. Hobert $\mathrm{O}$ : Gene regulation by transcription factors and microRNAs. Science 2008, 6:1785-1786.

56. Zhou Y, Ferguson J, Chang JT, Kluger Y: Inter- and intra-combinatorial regulation by transcription factors and microRNAs. BMC Genomics 2007, 6:396.

doi:10.1186/1472-6882-14-14

Cite this article as: Song et al:: Sho-saiko-to, a traditional herbal medicine, regulates gene expression and biological function by way of microRNAs in primary mouse hepatocytes. BMC Complementary and Alternative Medicine 2014 14:14

\section{Submit your next manuscript to BioMed Central and take full advantage of:}

- Convenient online submission

- Thorough peer review

- No space constraints or color figure charges

- Immediate publication on acceptance

- Inclusion in PubMed, CAS, Scopus and Google Scholar

- Research which is freely available for redistribution 